\title{
Motivating Developers to Use Interoperable Standards for Data in Pandemic Health Apps
}

\author{
Michael Rusongoza MUZOORA ${ }^{\mathrm{a}, \mathrm{b}, 1}$, Nabil EL-BADAWI ${ }^{\mathrm{a}, \mathrm{b}}$, Christian ELSNER $^{\mathrm{e}}$, \\ Andrea ESSENWANGER ${ }^{\mathrm{a}}$, Peter GOCKE ${ }^{\mathrm{b}}$, Dagmar KREFTING ${ }^{\mathrm{c}}$, Rasim Atakan \\ POYRAZ ${ }^{\mathrm{a}, \mathrm{b}}$, Rüdiger PRYSS ${ }^{\mathrm{f}}$, Ulrich SAX ${ }^{\mathrm{d}}$ and Sylvia THUN ${ }^{\mathrm{a}, \mathrm{b}, \mathrm{c}}$ \\ ${ }^{a}$ Berlin Institute of Health at Charité - Universitätsmedizin Berlin, Germany \\ ${ }^{b}$ Charité - Universitätsmedizin Berlin, Germany \\ ${ }^{\mathrm{c} H o c h s c h u l e ~ N i e d e r r h e i n}$ - University of Applied Sciences, Krefeld, Germany \\ ${ }^{\mathrm{d}}$ Universitätsmedizin Göttingen, Germany \\ e Universitätsmedizin Mainz, Germany \\ ${ }^{\mathrm{f}}$ Universitätsklinikum Würzburg, Germany
}

\begin{abstract}
The COVID-19 pandemic has brought along a massive increase in app development. However, most of these apps are not using interoperable data. The COMPASS project of the German COVID-19 Research Network of University Medicine ("Netzwerk Universitätsmedizin (NUM)") tackles this issue, by offering open-source technology, best practice catalogues, and suggestions for designing interoperable pandemic health applications (https://www.netzwerkuniversitaetsmedizin.de/projekte/compass). Therefore, COMPASS conceived a framework that includes automated conformity checks as well as reference implementations for more efficient and pandemic-tailored app developments. It further aims to motivate and support developers to use interoperable standards.
\end{abstract}

Keywords. Pandemic app, COVID-19, interoperability, healthcare data

\section{Introduction}

During the COVID-19 pandemic, the smartphone became the central way of sharing health related data [1]. A variety of pandemic apps were developed, but for researchers, to use the gathered data efficiently, the data needs to be interoperable and standardized [2]. One of the COMPASS project's main purposes is to establish a coordination and technology platform to ensure the use of standardized data and compliance with law and data protection regulations in the development of pandemic apps. This paper highlights the project's potential to motivate developers to use interoperable standards, such as FHIR, SNOMED CT, LOINC, for data in pandemic apps.

\footnotetext{
${ }^{1}$ Michael Rusongoza Muzoora, Berlin Institute of Health, Anna-Louisa-Karsch-Straße 2, 10178 Berlin, Germany; E-mail: michael.muzoora@charite.de.
} 


\section{Methods}

To have a better understanding of the project, we looked at the available documentation and websites. Additionally, we exchanged information with domain experts around the COMPASS Project, to incorporate SNOMED CT, FHIR as well as standardized datasets such as the GECCO (German Corona Consensus) dataset [3].

\section{Results}

In the open-source COMPASS project, a framework with ready-to-use app components will be developed. The framework includes interfaces, questionnaires, templates for privacy statements and terms of use, automated conformity checks as well as reference implementations [1]. Moreover, an automated seal of approval will be issued if the conformity check approves the validity towards interoperable datasets.

The COMPASS platform shall offer developers and researchers an easy way to use existing standards for COVID-19 data, such as the GECCO dataset, while also taking organizational, regulatory, and ethical aspects of the development process into consideration. A knowledge database (https://num.umg.eu/) taking these considerations into account is being established.

\section{Discussion and Conclusion}

In this article, we described how the COMPASS project motivates developers to work with medical data related to the current pandemic. The project aims to support and motivate developers to use interoperable data by simplifying the implementation as well as providing additional benefits. To avoid non-interoperable solutions, it is important to promote COMPASS to motivate developers to access the same resources.

\section{Acknowledgements}

The COMPASS project is part of the German COVID-19 Research Network of University Medicine ("Netzwerk Universitätsmedizin"), funded by the German Federal Ministry of Education and Research (funding reference 01KX2021).

\section{References}

[1] Profile | COMPASS [Internet]. num-compass.science. Universitätsmedizin der Johannes GutenbergUniversität Mainz; 2020 Available at:: https://num-compass.science/en/project/profile/, Accessed January 14, 2021.

[2] O'Reilly-Shah VN, Gentry KR, Van Cleve W, Kendale SM, Jabaley CS, Long DR. The COVID-19 Pandemic Highlights Shortcomings in US Health Care Informatics Infrastructure: A Call to Action. Anesth Analg. 2020 Aug;131(2):340-344.

[3] Sass, J., Bartschke, A., Lehne, M. et al. The German Corona Consensus Dataset (GECCO): a standardized dataset for COVID-19 research in university medicine and beyond. BMC Med Inform Decis Mak 2020;20:341. 